\title{
Modification of the spontaneous emission rate of nitrogen-vacancy centers in diamond by coupling to plasmons
}

\author{
Andrei Faraon ${ }^{1 *}$, Young Chul Jun ${ }^{2}$, Paul E. Barclay ${ }^{1}$, Kai-Mei C. Fu ${ }^{1}$, Charles M. Santori ${ }^{1}$, \\ Mark L. Brongersma ${ }^{2}$, Raymond G. Beausolei ${ }^{1}$ \\ ${ }^{1}$ Hewlett Packard Laboratories, 1501 Page Mill Rd., Palo Alto, CA, 94304, USA \\ ${ }^{2}$ Department of Materials Science and Engineering, Stanford University, Stanford, CA, \\ 94305,USA
}

\begin{abstract}
Nitrogen-vacancy centers in diamond are widely studied both as a testbed for solid state quantum optics and for their applications in quantum information processing and magnetometry. Here we demonstrate coupling of the nitrogen-vacancy centers to gap plasmons in metal nano-slits. We use diamond samples where nitrogen-vacancy centers are implanted tens of nanometers under the surface. Silver nano-slits are patterned on the sample such that diamond ridges tens of nanometers wide fill the slit gap. We measure enhancement of the spontaneous emission rate of the zero photon line by a factor of 3 at a temperature of $8 \mathrm{~K}$.
\end{abstract}

Keywords: quantum information, plasmon, diamond, nitrogen-vacancy center

\section{INTRODUCTION}

Large-scale quantum photonic circuits could enable applications that are impossible to realize on any classical computer, such as fundamentally secure communication, ${ }^{1}$ efficient factoring algorithms, ${ }^{2}$ and simulation of large quantum systems. ${ }^{3}$ Because of their spin properties, nitrogen vacancy (NV) centers in diamond are one of the most promising quantum optical emitters for integration in quantum networks. Key experiments on optical control of the spin states in nitrogen-vacancy centers, ${ }^{4,5}$ and entanglement between the state of a single nitrogen-vacancy and a single photon have already been demonstrated. ${ }^{6}$ One of the remaining challenges is to integrate the nitrogen-vacancy centers in an on-chip photonic network. This requires coupling to nanophotonic devices with an optical mode that can be further coupled into the network. Coupling has been observed using diamond nanoparticles, but the nitrogen-vacancies in these structures generally suffer from bad spectral properties. $^{7-12}$ The efforts for nanophotonic integration of nitrogen-vacancies located in single crystal diamond have been hindered so far by the difficulties with single crystal diamond fabrication ${ }^{13,14}$ or integration of diamond with other material systems that are easier to fabricate. ${ }^{15,16}$ Here we demonstrate coupling of nitrogen-vacancy centers to gap plasmons metal-insulator-metal (MIM) waveguides fabricated in single crystal diamond. Similar results have been observed using colloidal quantum dots coupled to metal nanoslits. ${ }^{17}$

\section{DEVICE FABRICATION AND CHARACTERIZATION}

A schematic of the device is shown in Fig. 1(a). It consists of diamond ridge (width $w$ and height $H$ ) in between two diamond slabs. This structure behaves like a metal-insulator-metal plasmonic waveguide. Two dimensional finite difference time domain (FDTD) simulations indicate that for a fixed ridge width $(w=50 \mathrm{~nm})$, the spontaneous emission rate enhancement $\left(\gamma / \gamma_{0}\right)$ for an optical dipole located in the middle of the ridge and oriented perpendicular to the metal facets has a strong dependence on $H$. Here $\gamma$ is the spontaneous emission rate inside the metal-insulator-metal waveguide and $\gamma_{0}$ is the spontaneous emission rate in bulk diamond. For an operating wavelength of $637 \mathrm{~nm}$, corresponding to the zero photon line of the negatively charged nitrogenvacancy centers, the maximum enhancement is obtained for $H \approx 120 \mathrm{~nm}$. Our simulations indicate that the enhancement becomes higher for smaller waveguide widths. However, as the slit width reaches the nano-meter range, non-radiative recombination may become the dominant process.

\footnotetext{
* To whom correspondence should be addressed. Email: andrei.faraon@hp.com
}

Advances in Photonics of Quantum Computing, Memory, and Communication IV, edited by Zameer UI Hasan, Philip R. Hemmer, Hwang Lee, Charles M. Santori, Proc. of SPIE Vol. 7948,794814 - (c) 2011 SPIE · CCC code: 0277-786X/11/\$18 - doi: 10.1117/12.874221 
(a)

$\operatorname{Air}(n=1)$

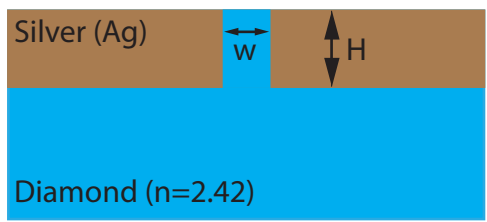

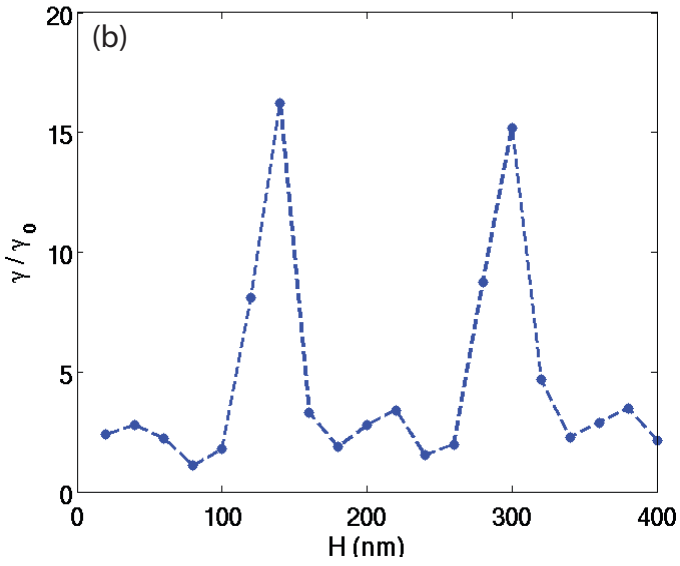

Figure 1. (a) Schematic of the simulated structure consisting of a diamond ridge of width $w$ and height $H$ in between two silver (Ag) slabs. (b) Simulated (FDTD) spontaneous emission rate enhancement as a function of ridge height for a fixed width $w=50 \mathrm{~nm}$

1. Diamond Sample

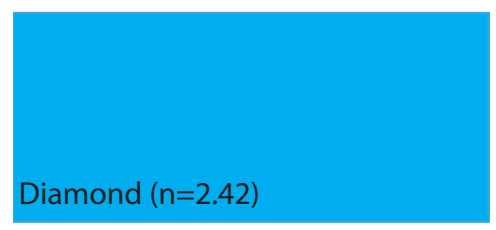

4. Etch ridges in diamond

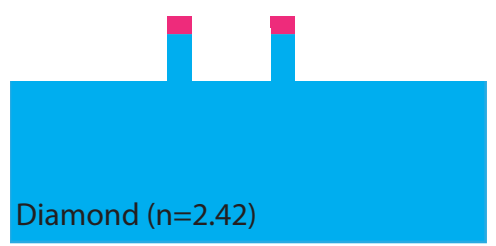

2. Spin Fox 12
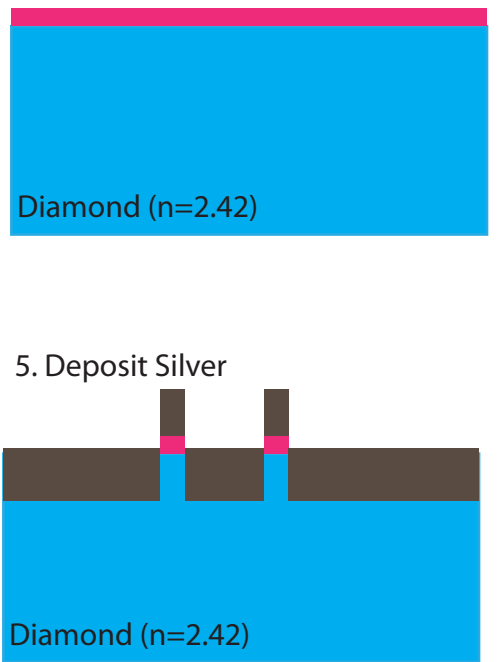

3. Pattern ridges using e-beam

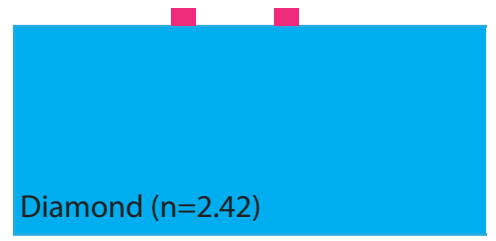

6. Liftoff

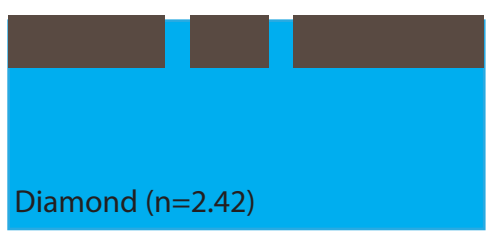

Figure 2. Schematic representation of the sequence of fabrication steps. 

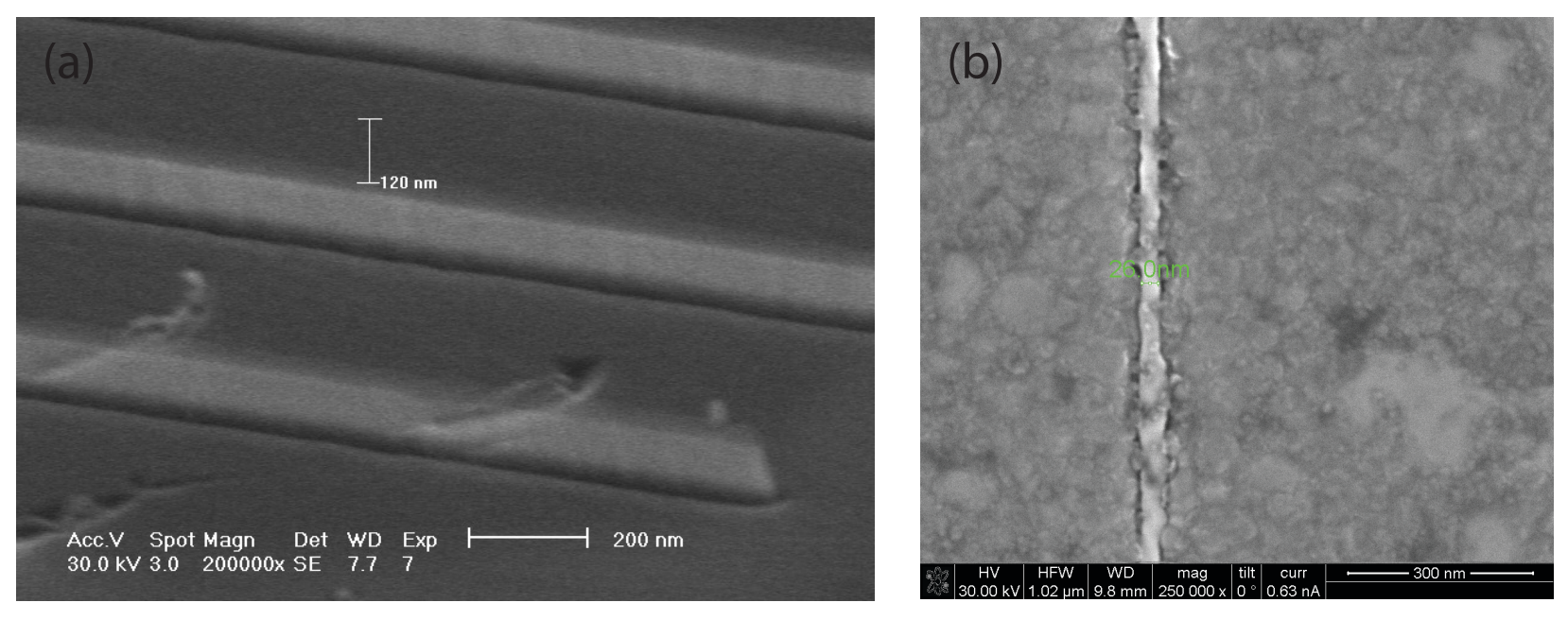

Figure 3. (a) Fabricated diamond ridges shown after the etching (120nm deep) step. (b) Top view of the ridge after silver deposition and liftoff.

The waveguides were fabricated in single crystal CVD diamond (Element 6). We use diamond samples where nitrogen-vacancy centers are implanted at an accelerating voltage of $50 \mathrm{KeV}$ and implantation density of $2 \times 10^{13} / \mathrm{cm}^{2}$. The nitrogen-vacancy centers can reach implantation depths as deep as $100 \mathrm{~nm}$, with the maximum concentration at a depth of $60 \mathrm{~nm}$, as determined from simulations.

Electron-beam lithography and and reactive ion etching were using in the fabrication process (Fig. 2). First, negative e-beam resist (FOX-12) was spun on the sample and the ridges were lithographically patterned. An oxygen plasma etching step (Oxford reactive ion-etching machine) was then used to etch 120nm into the diamond sample thus obtaining thin (tens of $\mathrm{nm}$ ) diamond ridges as shown in Fig. 3(a). Then 120nm silver was deposited on the sample and a liftoff procedure was used to remove the silver from the top of the ridges. A top image of the device is shown in Fig. 3(b). The diamond can be observed in between the silver slabs. The measurements done using the scanning electron microscope indicate that the top of the ridge has a thickness slightly smaller than $30 \mathrm{~nm}$. However, the etching profile of the ridge is not perfectly vertical. We used an FIB to determine that the thickness of the width of the ridge increases up to $\approx 60 \mathrm{~nm}$ at its bottom.

The sample characterization was done in a continuous flow He cryostat operating at 8K. A confocal microscope setup was used both for excitation and for collection of the photoluminescence signal. The spectrum of the NV centers under continuous wave green (532nm) excitation is shown in Fig. 4(a). This sample exhibits a pronounced zero phonon line at $637 \mathrm{~nm}$ that corresponds to the negatively charged nitrogen vacancy centers, and another zero phonon line at $575 \mathrm{~nm}$ that corresponds to the neutral nitrogen-vacancy centers. From the relative intensity of the zero phonon lines, it can be concluded that the nitrogen-vacancies in this sample are mainly negatively charged.

The lifetime of the NVs coupled to the metallic slit was determined from the photoluminescence signal under pulse excitation. For this measurement we used pulsed green light obtained by filtering a super continuum source. The super continuum generation was done in a photonic crystal fiber using laser pulses from a Ti:Sapphire laser. The average intensity of the pulsed light source was $200 \mu \mathrm{W}$. A grating filter, was used to filter the signal from the negatively charged zero phonon line and the output was sent to a avalanche silicon photodiode detector.

The experimental data both from nitrogen-vacancies in the slit and nitrogen-vacancies in bulk diamond is shown Fig. 4(b). The bulk lifetime data was acquired in ridges with large width (tens of microns), such that 
(a)

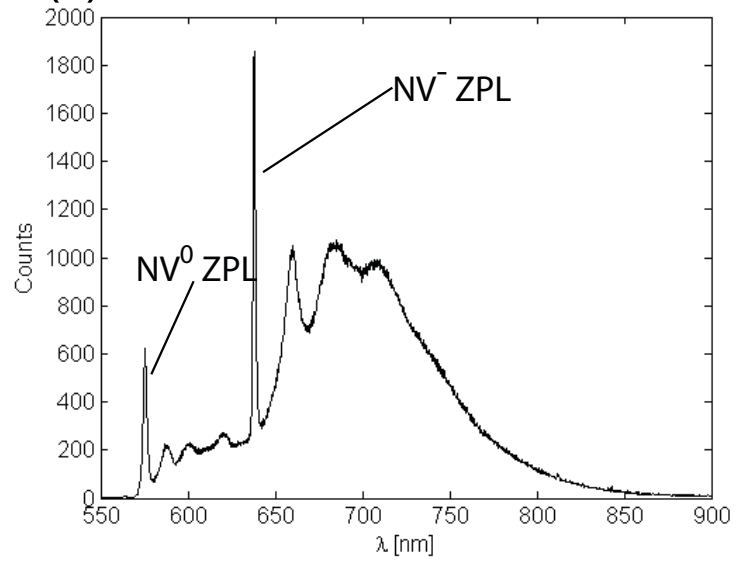

(b)

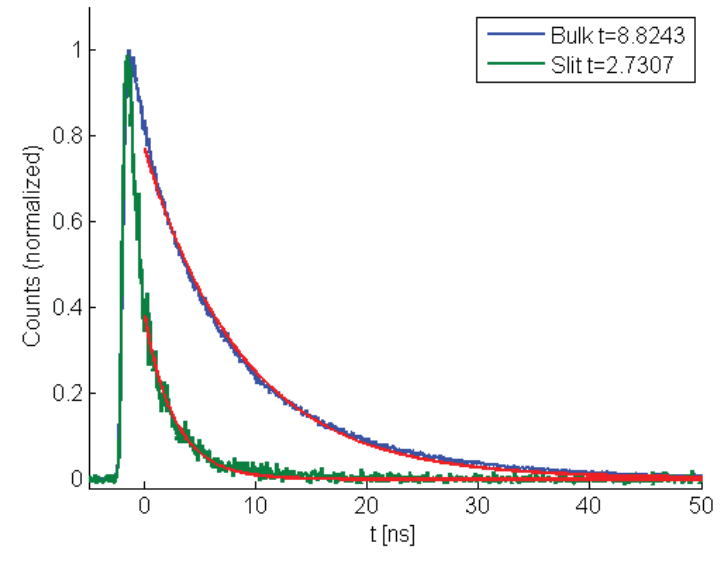

Figure 4. (a) Photoluminescence spectrum of an ensemble of NV centers under green excitation.(b) Lifetime measurement indicating that the NV lifetime (measured on the zero phonon line) decreases from $\approx 9 \mathrm{~ns}$ to $\approx 3 \mathrm{~ns}$

there is no coupling to gap plasmons. A single exponential fit is used to determine that the lifetime decreases by a factor of $\approx 3$ from $8.8 \mathrm{~ns}$ (in bulk) to $2.7 \mathrm{~ns}$ (in the slit). The bulk lifetime is significantly smaller than the value reported in the literature for $\mathrm{NV}$ centers $(\approx 12 \mathrm{~ns})$. We observe this in samples that have been heavily implanted. The measured enhancement factor is smaller than the factor of 15 estimated in Fig. 1(b) because the NV centers span a variety of orientations and locations with respect to the slit, and because of imperfections in the device fabrications. These imperfections can be observed in Fig. 3(b), where the size of the metal roughness is a significant fraction of the slit width. More measurements (currently in progress), as polarization dependence and lifetime dependence on slit width, are needed for an exact quantification of the coupling between the NVs and the gap plasmon mode. Most importantly, it is necessary to determine how much of the lifetime reduction is caused by radiative versus non-radiative processes.

One of the next steps for this type of experiment is to use samples with lower concentration of nitrogenvacancy centers, so the lifetime modification of single nitrogen-vacancy centers can be measured. This can be done in electronic-grade diamond sample. The fabrication process needs to be improved in order to obtain smooth metal surfaces that reduce the scattering loss. Significant scattering is in fact useful for experiments where the samples are probed from the top because more signal can be collected. However, the idea is to use the well defined mode of the slit waveguides for further coupling into optical networks. Also, once good NV coupling is obtained, one of the next steps is to do coherent probing measurements by coupling laser beams into the slit waveguide.

\section{CONCLUSION}

In conclusion, we demonstrate modification of the spontaneous emission rate for nitrogen-vacancy centers coupled to gap plasmons in silver nanoslits. The gap plasmon modes can be further coupled into a nano-photonic optical network, which is relevant for future quantum photonic technologies based on nitrogen-vacancy centers.

Other possibilities for coupling nitrogen-vacancy centers into photonic networks is to use dielectric resonators and waveguides. The dielectric resonators have high quality factors, which allows for resonant enhancement of 
the zero-phono line with respect to the phonon sidebands as recently demonstrated ${ }^{18}$ using ring resonators in single crystal diamond.

\section{REFERENCES}

1. L. Childress, J. M. Taylor, A. S. Sorensen, and M. D. Lukin. Fault-tolerant quantum repeaters with minimal physical resources and implementations based on single-photon emitters. Phys. Rev. A, 72:52330, 2005.

2. M. A. Nielsen and I. L. Chuang. Quantum Computation and Quantum Information. Cambridge University Press, Cambridge, 2000.

3. B. P. Lanyon, J. D. Whitfield, G. G. Gillett, M. E. Goggin, M. P. Almeida, I. Kassal, J. D. Biamonte, M. Mohseni, B. J. Powell, M. Barbieri, A. Aspuru-Guzik, and A. G. White. Towards quantum chemistry on a quantum computer. Nature Chem., 2(2):106-111, February 2010.

4. Charles Santori, Philippe Tamarat, Philipp Neumann, Jorg Wrachtrup, David Fattal, Raymond G. Beausoleil, James Rabeau, Paolo Olivero, Andrew D. Greentree, Steven Prawer, Fedor Jelezko, and Philip Hemmer. Coherent population trapping of single spins in diamond under optical excitation. Phys. Rev. Lett., 97(24):247401, 2006.

5. B. B. Buckley, G. D. Fuchs, L. C. Bassett, and D. D. Awschalom. Spin-Light Coherence for Single-Spin Measurement and Control in Diamond. Science, 330(6008):1212-1215, 2010.

6. E. Togan, Y. Chu, A. S. Trifonov, L. Jiang, J. Maze, L. Childress, M. V. G. Dutt, A. S. Sorensen, P. R. Hemmer, A. S. Zibrov, and M. D. Lukin. Quantum entanglement between an optical photon and a solid-state spin qubit. Nature, 466(7307):730-734, August 2010.

7. Stefan Schietinger, Michael Barth, Thomas Aichele, and Oliver Benson. Plasmon-enhanced single photon emission from a nanoassembled metal-diamond hybrid structure at room temperature. Nano Letters, 9(4):1694-1698, 2009. PMID: 19301860.

8. Paul E. Barclay, Charles Santori, Kai-Mei Fu, Raymond G. Beausoleil, and Oskar Painter. Coherent interference effects in a nano-assembled diamond nv center cavity-qed system. Opt. Express, 17(10):8081-8097, 2009.

9. Y.-S. Park, A. K. Cook, and H. Wang. Cavity qed with diamond nanocrystals and silica microspheres. Nano Lett., 6(9):2075-2079, 2006.

10. Janik Wolters, Andreas W. Schell, Gunter Kewes, Nils Nusse, Max Schoengen, Henning Doscher, Thomas Hannappel, Bernd Lochel, Michael Barth, and Oliver Benson. Enhancement of the zero phonon line emission from a single nitrogen vacancy center in a nanodiamond via coupling to a photonic crystal cavity. Applied Physics Letters, 97(14):141108, 2010.

11. Dirk Englund, Brendan Shields, Kelley Rivoire, Fariba Hatami, Jelena Vuckovic, Hongkun Park, and Mikhail D. Lukin. Deterministic coupling of a single nitrogen vacancy center to a photonic crystal cavity. Nano Letters, 10(10):3922-3926, 2010.

12. T. van der Sar, J. Hagemeier, W. Pfaff, E.C. Heeres, T.H. Oosterkamp, D. Bouwmeester, and R. Hanson. Deterministic nano-assembly of a coupled quantum emitter - photonic crystal cavity system. arXiv:1008.4097v1 [quant-ph], 2010.

13. M.P. Hiscocks, K. Ganesan, B.C. Gibson, S.T. Huntington, F. Ladouceur, and S. Prawer. Diamond waveguides fabricated by reactive ion etching. Opt. Express, 16(24):19512-19519, 2008.

14. T. M. Babinec, J. T. Choy, K. J. M. Smith, M. Khan, and M. Loncar. Design and focused ion beam fabrication of single crystal diamond nanobeam cavities. arXiv:1008.1431v2 [quant-ph], 2010.

15. K.-M. C. Fu, C. Santori, P. E. Barclay, I. Aharonovich, S. Prawer, N. Meyer, A. M. Holm, and R. G. Beausoleil. Coupling of nitrogen-vacancy centers in diamond to a gap waveguide. Appl. Phys. Lett., 93(23):234107, 2008.

16. Paul E. Barclay, Kai-Mei C. Fu, Charles Santori, and Raymond G. Beausoleil. Chip-based microcavities coupled to nitrogen-vacancy centers in single crystal diamond. Appl. Phys. Lett., 95(19):191115, 2009.

17. Young Chul Jun, Ragip Pala, and Mark L. Brongersma. Strong modification of quantum dot spontaneous emission via gap plasmon coupling in metal nanoslits. The Journal of Physical Chemistry C, 114(16):7269$7273,2010$.

18. Andrei Faraon, Paul E. Barclay, Charles Santori, Kai-Mei C. Fu, and Raymond G. Beausoleil. Resonant enhancement of the zero-phonon emission from a color center in a diamond cavity. arXiv:1012.3815v1 [quant-ph], 2010. 